\title{
Global rigidity of direction-length frameworks
}

\author{
Katie Clinch* $\quad$ Bill Jackson ${ }^{\dagger} \quad$ Peter Keevash $^{\ddagger}$
}

May 9, 2020

\begin{abstract}
A 2-dimensional direction-length framework is a collection of points in the plane which are linked by pairwise constraints that fix the direction or length of the line segments joining certain pairs of points. We represent it as a pair $(G, p)$, where $G=(V ; D, L)$ is a 'mixed' graph and $p: V \rightarrow \mathbb{R}^{2}$ is a point configuration for $V$. It is globally rigid if every direction-length framework $(G, q)$ which satisfies the same constraints can be obtained from $(G, p)$ by a translation or a rotation by $180^{\circ}$. We characterise the mixed graphs $G$ with the property that every generic framework $(G, p)$ is globally rigid.
\end{abstract}

\section{Introduction}

A finite configuration of points in Euclidean space with local constraints may be informally described as globally rigid if the constraints determine the point set up to congruence. It is a fundamental open problem to give a nice characterisation of global rigidity in various settings. Our setting here is that of a $d$-dimensional direction-length framework, which is a pair $(G, p)$, where $G=(V ; D, L)$ is a 'mixed' graph and $p: V \rightarrow \mathbb{R}^{d}$ is a point configuration for $V$. (We will be particularly concerned with the case when $d=2$.) We call the graph $G$ mixed because it has two types of edges: we refer to edges in $D$ as direction edges and edges in $L$ as length edges. The graph may contain parallel edges as long as they are of different types. Two direction-length frameworks $(G, p)$ and $(G, q)$ are equivalent if $p(u)-p(v)$ is a scalar multiple of $q(u)-q(v)$ for all $u v \in D$ with $q(u) \neq q(v)$, and $\|p(u)-p(v)\|=\|q(u)-q(v)\|$ for all $u v \in L$. Two point configurations $p$ and $q$ for $V$ are congruent if either $p(u)-p(v)=q(u)-q(v)$ for all $u, v \in V$, or $p(u)-p(v)=q(v)-q(u)$ for all $u, v \in V$. (Thus $p$ and $q$ are congruent if $p$ can be obtained from $q$ by a translation, possibly followed

\footnotetext{
* School of Mathematical Sciences, Queen Mary, University of London, Mile End Road, London E1 4NS, UK. Email: k.clinch@qmul.ac.uk

${ }^{\dagger}$ School of Mathematical Sciences, Queen Mary, University of London, Mile End Road, London E1 4NS, UK. Email: b.jackson@qmul.ac.uk

${ }^{\ddagger}$ Mathematical Institute, University of Oxford, Woodstock Road, Oxford OX2 6GG, UK. Email: keevash@maths.ox.ac.uk.
} 

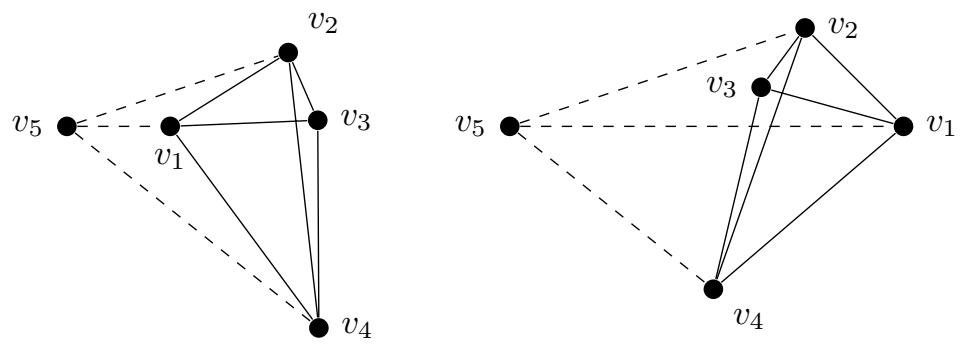

Figure 1: Two equivalent but non-congruent direction-length frameworks. We use solid or dashed lines to indicate length or direction constraints, respectively. The frameworks are rigid but not globally rigid. They are not redundantly rigid since they cease to be rigid if we delete one of the direction constraints - this will allow us to rotate the subgraph induced by the length edges and adjust the position of the vertex $v_{5}$ to preserve the remaining two direction constraints.

by a rotation by $180^{\circ}$.) A direction-length framework $(G, p)$ is globally rigid if $p$ is congruent to $q$ for every framework $(G, q)$ which is equivalent to $(G, p)$. It is rigid if there exists an $\varepsilon>0$ such that, for every framework $(G, q)$ which is equivalent to $(G, p)$ and satisfies $\|p(v)-q(v)\|<\varepsilon$ for all $v \in V$, we have $p$ is congruent to $q$. (Equivalently every continuous motion of the vertices of $(G, p)$ which satisfies the direction and length constraints given by the edges results in a framework $(G, q)$ with $p$ congruent to $q$.) The framework $(G, p)$ is redundantly rigid if $(G-e, p)$ is rigid for all $e \in D \cup L$. These concepts are illustrated in Figure 1.

We will consider generic frameworks, meaning that the set containing the coordinates of all of the vertices is algebraically independent over the rationals; this eliminates many pathologies. It follows from $[7,8]$ that rigidity is a 'generic property' in the sense that if some realisation of a mixed graph $G$ as a generic framework in $\mathbb{R}^{d}$ is rigid then all generic realisations of $G$ in $\mathbb{R}^{d}$ are rigid. This implies that redundant rigidity is also a generic property and allows us to describe a mixed graph $G$ as being rigid or redundantly rigid in $\mathbb{R}^{d}$ if some (or equivalently if every) generic realisation of $G$ has these properties. It is not known whether global rigidity is a generic property (however this statement would follow from Conjecture 9.1 below in the 2-dimensional case).

Both rigidity and global rigidity are known to be generic properties for $d$ dimensional pure frameworks, i.e. frameworks which contain only length constraints or only direction constraints. We will not give formal definitions for rigidity and global rigidity of pure frameworks - they are similar to those for direction-length frameworks except that the notion of congruence will allow not only translations, but also arbitrary rotations and reflections in the case of length-pure frameworks, and dilations in the case of direction-pure frameworks.

The problems of characterising rigidity and global rigidity for 2-dimensional generic length-pure frameworks were solved by Laman [10] and Jackson and 
Jordán [5], respectively. In particular, [5] proved that a 2-dimensional generic length-pure framework $(G, p)$ is globally rigid if and only if either $G$ is a complete graph on at most 3 vertices, or $G$ is 3-connected and redundantly rigid. The problems of characterising rigidity and global rigidity for $d$-dimensional generic length-pure frameworks are open for $d \geq 3$. In contrast, Whiteley [16] characterised $d$-dimensional generic direction-pure frameworks which are rigid for all $d$. He also observed that the linearity of direction constraints implies

Lemma 1.1. [16] Suppose that $(G, p)$ is a direction-pure framework. Then $(G, p)$ is globally rigid if and only if $(G, p)$ is rigid.

Since the problem of characterising generic rigidity of direction-length frameworks is at least as hard as that for length-pure frameworks, we will henceforth restrict our attention to 2-dimensional frameworks. Rigid generic (2dimensional) direction-length frameworks were characterised by Servatius and Whiteley in [14]. They also pointed out that Lemma 1.1 gives the following characterisation of global rigidity for generic direction-length frameworks with exactly one length constraint.

Lemma 1.2. [14] Suppose that $(G, p)$ is a generic realisation of a mixed graph $G=(V ; D, L)$ with $|L|=1$. Then $(G, p)$ is globally rigid if and only if $G$ is rigid.

Further results on global rigidity were obtained by Jackson and Jordán in [6] who showed that two necessary conditions for a generic 2-dimensional directionlength framework $(G, p)$ to be globally rigid are that $G$ is 2-connected and direction-balanced i.e. whenever $H_{1}, H_{2}$ are subgraphs of $G$ with $G=H_{1} \cup H_{2}$, $V\left(H_{1}\right) \cap V\left(H_{2}\right)=\{u, v\}$ and $V\left(H_{1}\right) \backslash V\left(H_{2}\right) \neq \emptyset \neq V\left(H_{2}\right) \backslash V\left(H_{1}\right)$, both $H_{1}$ and $H_{2}$ must contain a direction edge of $G$ distinct from uv. They also showed that these conditions are sufficient when $G$ is redundantly rigid and has exactly $2|V|-1$ edges.

We will see in Section 2.1 that we may define a matroid $M(G)$ on the edge set of a mixed graph $G$ in such a way that $G$ is rigid if and only if $M(G)$ has rank $2|V|-2$. The above sufficient condition for global rigidity, that $G$ is redundantly rigid and has $2|V|-1$ edges, is equivalent to the edge set of $G$ being a rigid circuit of $M(G)$. A mixed graph $G$ is redundantly rigid if and only if it is rigid and every edge of $G$ is contained in a circuit of $M(G)$. We say that $G$ is $M$-connected if it satisfies the stronger condition that every pair of edges of $G$ is contained in a circuit of $M(G)$ i.e. $M(G)$ is a connected matroid. Clinch [2] has recently shown that the above mentioned necessary conditions for generic global rigidity are also sufficient when the underlying mixed graph is $M$-connected and rigid.

Theorem 1.3. Suppose $(G, p)$ is a generic realisation of an $M$-connected rigid mixed graph $G$. Then $(G, p)$ is globally rigid if and only if $G$ is directionbalanced.

Unfortunately, Clinch's result does not give a complete characterisation of generic global rigidity because $M$-connectivity is not a necessary condition for 
the global rigidity of generic rigid frameworks. This follows from the above mentioned fact that every generic realisation of a (minimally) rigid mixed graph with exactly one length edge is globally rigid, or from the fact that global rigidity is preserved if we join a new vertex to an existing globally rigid framework by two direction constraints. (The underlying graphs in both constructions are not even redundantly rigid.) We can generalise the second construction as follows.

Suppose $(G, p)$ is a generic realisation of a mixed graph $G$ which has a proper induced subgraph $H$ such that the graph $G / H$ obtained from $G$ by contracting $H$ to a single vertex (deleting all edges of $H$ but keeping all other edges of $G$, possibly as parallel edges) has only direction edges and is the union of two edge-disjoint spanning trees. We will see in Section 4 that $G-e$ is not rigid for all direction edges $e$ which do not belong to $H$ (hence $G$ is not redundantly rigid), and that $(G, p)$ is globally rigid if and only if $\left(H,\left.p\right|_{H}\right)$ is globally rigid.

These observations lead us to consider a more general reduction operation for a mixed graph $G$. We say that $G$ admits a direction reduction to a subgraph $H$ if either:

(R1) $H=G-e$ for some edge $e \in D$ which belongs to a direction-pure circuit in the rigidity matroid of $G$, or

(R2) $H$ is a proper induced subgraph of $G$, and $G / H$ is direction-pure and is the union of two edge-disjoint spanning trees.

If $G$ has no direction reduction, then we say that $G$ is direction irreducible. (We will describe an efficient algorithm in Section 8 which either finds a direction reduction of a given mixed graph or concludes that it is direction irreducible.) An example of a direction reduction is given in Figure 2.

Our first result reduces the problem of characterising the global rigidity of a generic framework $(G, p)$ to the case when $G$ is direction irreducible.

Theorem 1.4. Suppose $(G, p)$ is a generic direction-length framework and $G$ admits a direction reduction to a subgraph $H$. Then $(G, p)$ is globally rigid if and only if $\left(H,\left.p\right|_{H}\right)$ is globally rigid.

We will obtain structural information about the family of direction irreducible mixed graphs which are not redundantly rigid, and have at least two length edges. We use this information to characterise when such graphs are globally rigid for all generic realisations:

Theorem 1.5. Suppose $G$ is a direction irreducible mixed graph with at least two length edges. Then every generic realisation of $G$ is globally rigid if and only if $G$ is 2-connected, direction-balanced and redundantly rigid.

This leads to our main result, a characterisation of the direction-length graphs which are globally rigid for all generic realisations:

Theorem 1.6. Suppose $G=(V ; D, L)$ is a mixed graph. Then all generic realisations of $G$ are globally rigid if and only if $G$ is rigid, and either $|L|=1$ or $G$ has a direction-balanced, $M$-connected mixed subgraph which contains all edges in $L$. 

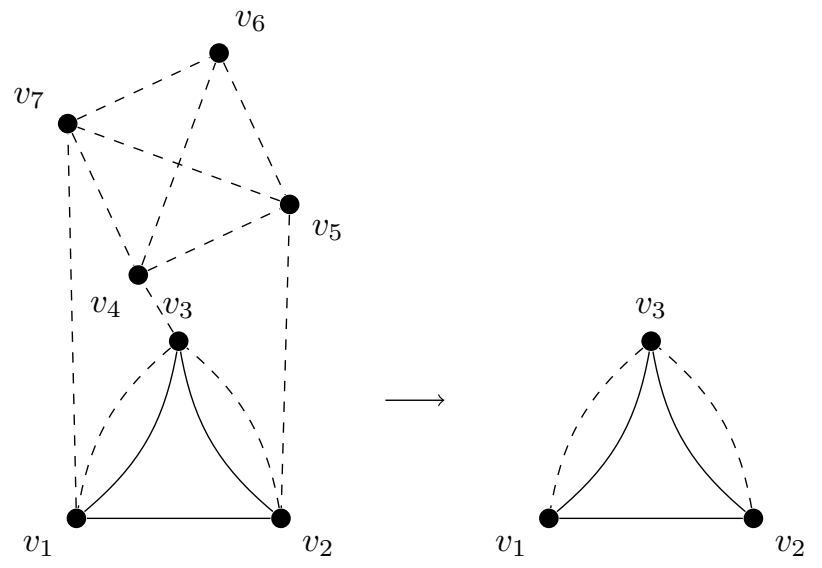

Figure 2: The graph $G$ on the left is direction reducible to the subgraph $H$ on the right in two steps. Since the direction edge $v_{5} v_{7}$ is contained in the directionpure circuit induced by $\left\{v_{4}, v_{5}, v_{6}, v_{7}\right\}$ we can delete $v_{5} v_{7}$ by (R1). The graph we now obtain by contracting $H$ to a single vertex is direction-pure and is the union of two edge-disjoint spanning trees so we can reduce $G$ to $H$ by (R2). Theorem 1.4 now tells us that a generic framework $(G, p)$ is globally rigid if and only if $\left(H,\left.p\right|_{H}\right)$ is globally rigid. Since $H$ is $M$-connected and rigid, $\left(H,\left.p\right|_{H}\right)$ is globally rigid by Theorem 1.3. Hence $(G, p)$ is globally rigid.

The organisation of this paper by section is 1: Introduction, 2: Preliminaries, 3: Realisations of graphs with given direction constraints, 4: Direction reductions and proof of Theorem 1.4, 5: Direction irreducible graphs, 6: Proof of Theorem 1.5, 7: Proof of Theorem 1.6, 8: Algorithmic considerations, 9: Closing remarks.

\section{Preliminaries}

In this section we collect tools from diverse areas that we will use in our proofs.

\section{$2.1 \quad$ Rigidity}

Suppose $(G, p)$ is a 2-dimensional direction-length framework. Its rigidity matrix is a $(|D|+|L|) \times 2|V|$ matrix $R(G, p)$, where each edge in $D \cup L$ corresponds to a row and each vertex in $V$ corresponds to a pair of consecutive columns. We choose an arbitrary reference orientation for the edges, and use the notation $e=u v$ to mean that $e$ has been oriented from $u$ to $v$. Fix an edge $e=u v$, a vertex $x$, and write $p(u)-p(v)=(a, b)$. Then the two entries in the rigidity matrix corresponding to $e$ and $x$ are as follows. If $e \in L$ we take $(a, b)$ if $x=u$, $(-a,-b)$ if $x=v,(0,0)$ otherwise. If $e \in D$ we take $(b,-a)$ if $x=u,(-b, a)$ if $x=v,(0,0)$ otherwise. 
We refer to vectors in the null space $Z(G, p)$ of $R(G, p)$ as infinitesimal motions of $(G, p)$. The labeling of the columns of $R(G, p)$ allows us to consider each infinitesimal motion $z$ as a map from $V$ to $\mathbb{R}^{2}$, with the properties that $z(u)-z(v)$ is perpendicular to $p(u)-p(v)$ if $e=u v \in L$, or parallel to $p(u)-p(v)$ if $e=u v \in D$. For any $t \in \mathbb{R}^{2}$ the translation given by $z(v)=t$ for all $v \in V$ is an infinitesimal motion, so $\operatorname{dim} Z(G, p) \geq 2$ and $\operatorname{rank} R(G, p) \leq 2|V|-2$. We say that the framework $(G, p)$ is infinitesimally rigid if $\operatorname{rank} R(G, p)=2|V|-2$, and is independent if the rows of $R(G, p)$ are linearly independent.

A property $\mathrm{P}$ of frameworks is generic if whenever some generic realisation of a graph $G$ has property $\mathrm{P}$ then all generic realisations of $G$ have property P. If $\mathrm{P}$ is a generic property then we say that $G$ has property $P$ if some generic realisation of $G$ has property P (or equivalently all generic realisations of $G$ have property $\mathrm{P}$ ). Infinitesimal rigidity and independence are both generic properties, as the rank of $R(G, p)$ is the same for all generic realisations of $G$. Results from $[7,8]$, which will be described in Section 2.5 , imply that infinitesimal rigidity and rigidity are equivalent properties for generic direction-length frameworks. Thus rigidity and redundant rigidity are also generic properties.

The rigidity matrix of $(G, p)$ defines the rigidity matroid of $(G, p)$ : the ground set $D \cup L$ corresponds to rows of the rigidity matrix, and a subset is independent when the corresponding rows are linearly independent. Any two generic realisations of $G$ have the same rigidity matroid, which we call the (2-dimensional) rigidity matroid $M(G)$ of $G$. (We refer the reader to [13] for an introduction to the theory of matroids.)

Servatius and Whiteley [14] characterised independence in the rigidity matroid of a mixed graph:

Theorem 2.1. Let $G=(V ; D, L)$ be a mixed graph and $F \subseteq D \cup L$. Then $F$ is independent in $M(G)$ if and only if for all $\emptyset \neq F^{\prime} \subseteq F$ we have $\left|F^{\prime}\right| \leq$ $2\left|V\left(F^{\prime}\right)\right|-2$, with strict inequality if $F^{\prime}$ is pure.

They also gave the following recursive construction for independent rigid mixed graphs, i.e. bases in the rigidity matroid of the 'complete mixed graph'. A 0 -extension of $G$ is a mixed graph obtained from $G$ by adding a new vertex $v$ and two edges at $v$, either of which may be a length edge or a direction edge, and which may go to the same vertex of $G$ if they consist of one length edge and one direction edge. A 1-extension of $G$ is a mixed graph obtained from $G$ by adding a new vertex $v$, deleting an edge $e$ of $G$, and adding three edges at $v$, such that the neighbours of $v$ include both endpoints of $e$, neither $D$ nor $L$ decrease in size, and two new edges may go to the same vertex if they are of different types. They showed that 0-extensions and 1-extensions preserve independence and rigidity, and conversely, any independent rigid mixed graph can be constructed starting from a single vertex by a sequence of 0 -extensions and 1-extensions. 


\section{$2.2 \quad M$-circuits and $M$-components}

An $M$-circuit of a mixed graph $G$ is a subgraph induced by the edges of a circuit of the rigidity matroid $M(G)$. Theorem 2.1 implies that $F$ is a circuit of $M(G)$ if and only if $F-e$ is independent for all $e \in F$ and either $F$ is mixed with $|F|=2|V(F)|-1$, or $F$ is pure with $|F|=2|V(F)|-2$. It also implies that mixed $M$-circuits are redundantly rigid. The characterisations of generic rigidity for pure frameworks similarly imply that length-pure $M$-circuits are redundantly length-rigid and direction-pure $M$-circuits are redundantly direction-rigid.

It is well known that a matroid can be expressed as the direct sum of its connected components, which are the equivalence classes of the relation $\sim$, where $e \sim f$ if $e=f$ or there is a circuit containing $e$ and $f$. We define the $M$ components of a mixed graph $G=(V ; D, L)$ to be the subgraphs induced by the edges in the connected components of its rigidity matroid $M(G)$. We can use the direct sum decomposition of the rigidity matroid $M(G)$ to calculate its rank, which we will denote by $r(G)$. Indeed, if $G$ has $M$-components $H_{1}, \ldots, H_{m}$ then we have $r(G)=\sum_{i=1}^{m} r\left(H_{i}\right)$, where $r\left(H_{i}\right)$ is $2\left|V\left(H_{i}\right)\right|-3$ when $H_{i}$ is pure and is $2\left|V\left(H_{i}\right)\right|-2$ otherwise. We can use this fact to show that $M$-connectivity is equivalent to redundant rigidity when $G$ is direction irreducible and satisfies the necessary conditions for generic global rigidity described in Section 1.

Lemma 2.2. Suppose $G$ is a direction irreducible, 2-connected, direction-balanced rigid mixed graph. Then $G$ is $M$-connected if and only if $G$ is redundantly rigid.

Proof. We have already seen, in Section 1 that redundant rigidity is a necessary condition for the $M$-connectivity of rigid mixed graphs. To prove sufficiency we suppose that $G$ is redundantly rigid but not $M$-connected. Let $H_{1}, H_{2}, \ldots, H_{m}$ be the $M$-components of $G$. Let $V_{i}=V\left(H_{i}\right), X_{i}=V_{i}-\bigcup_{j \neq i} V_{j}$ and $Y_{i}=V_{i}-X_{i}$ for all $1 \leq i \leq m$. Since $G$ is redundantly rigid, every edge of $G$ is contained in some $M$-circuit. Hence $\left|V_{i}\right| \geq 3$ for all $1 \leq i \leq m$. Since $G$ is 2 -connected, $\left|Y_{i}\right| \geq 2$ for all $1 \leq i \leq m$, and since $G$ is direction-balanced, $\left|Y_{i}\right| \geq 3$ when $H_{i}$ is length-pure. Since $G$ is direction irreducible, no direction edge of $G$ is contained in a direction-pure $M$-circuit. This implies that each of the $M$ connected components is either mixed or length-pure. Without loss of generality, we may assume that $H_{1}, H_{2}, \ldots, H_{\ell}$ are length-pure for some $0 \leq \ell \leq m$, and $H_{\ell+1}, H_{\ell+2}, \ldots, H_{m}$ are mixed. Then

$$
\begin{aligned}
r(G) & =\sum_{i=1}^{\ell}\left(2\left|V_{i}\right|-3\right)+\sum_{i=\ell+1}^{m}\left(2\left|V_{i}\right|-2\right) \\
& =\sum_{i=1}^{\ell}\left(2\left|X_{i}\right|+2\left|Y_{i}\right|-3\right)+\sum_{i=\ell+1}^{m}\left(2\left|X_{i}\right|+2\left|Y_{i}\right|-2\right) \\
& \geq \sum_{i=1}^{m}\left(2\left|X_{i}\right|+\left|Y_{i}\right|\right),
\end{aligned}
$$

since $\left|Y_{i}\right| \geq 3$ for all $1 \leq i \leq \ell$, and $\left|Y_{i}\right| \geq 2$ for all $\ell+1 \leq i \leq m$. Since the $X_{i}$ are all disjoint, we have $\sum_{i=1}^{m}\left|X_{i}\right|=\left|\bigcup_{i=1}^{m} X_{i}\right|$. Also, since each element of $Y_{i}$ 
is contained in at least one other $Y_{j}$ with $j \neq i$, we have $\sum_{i=1}^{m}\left|Y_{i}\right| \geq 2\left|\bigcup_{i=1}^{m} Y_{i}\right|$. Thus

$$
r(G) \geq 2\left(\left|\bigcup_{i=1}^{m} X_{i}\right|+\left|\bigcup_{i=1}^{m} Y_{i}\right|\right)=2|V| .
$$

This contradicts the fact that $r(G) \leq 2|V|-2$.

\subsection{Boundedness and global rigidity}

Now we recall some results from $[8,9]$. A direction-length framework $(G, p)$ is bounded if there exists a real number $K$ such that $\|q(u)-q(v)\|<K$ for all $u, v \in V$ whenever $(G, q)$ is a framework equivalent to $(G, p)$. It is known that the boundedness of $(G, p)$ is equivalent to the rigidity of an augmented framework, $\left(G^{+}, p\right)$.

Lemma 2.3. [8, Theorem 5.1] Let $(G, p)$ be a direction-length framework and let $G^{+}$be obtained from $G$ by adding a direction edge parallel to each length edge of $G$. Then $(G, p)$ is bounded if and only if $\left(G^{+}, p\right)$ is rigid.

Lemma 2.3 implies that boundedness is a generic property, and we say that a mixed graph $G$ is bounded if some, or equivalently every, generic realisation of $G$ is bounded. It also implies that every rigid mixed graph is bounded.

A mixed graph $G=(V ; D, L)$ is direction-independent if $D$ is independent in the direction-length rigidity matroid of $G$, i.e. the rows of $R(G, p)$ corresponding to $D$ are linearly independent for any generic $p$. Lemma 1.1 and the fact that direction-pure $M$-circuits are redundantly direction-rigid allow us to reduce the problem of deciding if a mixed graph is bounded to the family of directionindependent mixed graphs. The following characterisation of boundedness for direction-independent mixed graphs follows from [8, Theorem 5.1 and Corollary 4.3].

Lemma 2.4. Suppose that $G=(V ; D, L)$ is a direction-independent mixed graph. Then $G$ is bounded if and only if $G / L$ has two edge-disjoint spanning trees (where $G / L$ is the graph obtained from $G$ by contracting each edge in $L$ and keeping all multiple copies of direction edges created by this contraction).

A bounded component of $G$ is a maximal bounded subgraph of $G$. It is shown in [8] that each edge $e \in L$ lies in a bounded component and that the vertex sets of the bounded components partition $V$. We will use the following result of Nash-Williams [12] to show that there are relatively few edges between a set of bounded components in a mixed graph.

Theorem 2.5. The edge set of a graph $H$ can be covered by $k$ forests if and only if every non-empty set $X$ of vertices of $H$ induces at most $k|X|-k$ edges of $H$.

Lemma 2.6. Suppose $G=(V ; D, L)$ is direction-independent and $\mathcal{S}$ is a set of bounded components of $G$ with $|\mathcal{S}| \geq 2$. Then there are at most $2|\mathcal{S}|-3$ edges of $G$ joining distinct components in $\mathcal{S}$. 
Proof. Suppose on the contrary that there are at least $2|\mathcal{S}|-2$ edges of $G$ that join distinct components in $\mathcal{S}$. Suppose also that $\mathcal{S}$ is minimal with respect to this property (and the condition that $|\mathcal{S}| \geq 2$ ). Let $H$ be a graph with vertex set $\mathcal{S}$ and exactly $2|\mathcal{S}|-2$ edges, each of which correspond to a distinct edge of $G$ joining two components in $\mathcal{S}$. The minimality of $\mathcal{S}$ implies that every nonempty set $X$ of vertices of $H$ induces at most $2|X|-2$ edges of $H$ and hence, by Theorem 2.5, $H$ can be partitioned into two edge-disjoint spanning trees. By Lemma 2.4, for each bounded component $C_{i}=\left(V_{i} ; D_{i}, L_{i}\right) \in \mathcal{S}, C_{i} / L_{i}$ has two edge-disjoint spanning trees. We can combine the edge sets of these trees with the edge sets of the two edge-disjoint spanning trees of $H$ to obtain two edge-disjoint spanning trees in $G^{\prime} / L^{\prime}$, where $G^{\prime}=\left(V^{\prime} ; D^{\prime}, L^{\prime}\right)$ is the subgraph of $G$ induced by $\bigcup_{C_{i} \in \mathcal{S}} V\left(C_{i}\right)$. Lemma 2.4 now implies that $G^{\prime}$ is bounded and hence is contained in a single bounded component of $G$. This contradicts the fact that $|\mathcal{S}| \geq 2$.

We next state a result of [9] on global rigidity, which establishes that lengthredundancy is a necessary condition for generic global rigidity when $|L| \geq 2$, and takes a first step towards understanding when direction-redundancy is necessary. A subgraph of a mixed graph is said to be trivial if it has exactly one vertex, otherwise it is non-trivial.

Theorem 2.7. [9] Suppose that $(G, p)$ is a globally rigid generic realisation of a mixed graph $G=(V ; D, L)$ and $e$ is an edge of $G$.

(a) If $e \in L$ and $|L| \geq 2$ then $G-e$ is rigid.

(b) If $e \in D$ and $G-e$ has a non-trivial rigid subgraph then $G-e$ is either rigid or unbounded.

\subsection{Substitution}

The following subgraph substitution operation is an important tool which we will use throughout this paper. Suppose $G=(V ; D, L)$ is a mixed graph, $U \subseteq V$, $H=G[U]$ is the subgraph of $G$ induced by $U$, and $H^{\prime}$ is another mixed graph with vertex set $U$. Then the substitution $G^{\prime}$ of $H$ by $H^{\prime}$ in $G$ is obtained from $G$ by deleting all edges of $H$ and adding all edges of $H^{\prime}$. We record the following properties.

Lemma 2.8. If $G, H$ and $H^{\prime}$ are rigid then $G^{\prime}$ is rigid.

Proof. The ranks of $G$ and $G^{\prime}$ are both equal to the rank of the graph obtained from $G$ by joining all pairs of vertices of $H$ by both a direction and a length edge.

Lemma 2.9. Suppose $p: V \rightarrow \mathbb{R}^{2}$ is such that $(G, p)$ and $\left(H^{\prime},\left.p\right|_{U}\right)$ are both globally rigid. Then $\left(G^{\prime}, p\right)$ is globally rigid.

Proof. Let $\left(G^{\prime}, q\right)$ be an equivalent framework to $\left(G^{\prime}, p\right)$. Since $\left(H^{\prime},\left.p\right|_{U}\right)$ is globally rigid, $\left.q\right|_{U}$ is congruent to $\left.p\right|_{U}$. In particular, $\left(H,\left.q\right|_{U}\right)$ and $\left(H,\left.p\right|_{U}\right)$ are equivalent. But $G$ and $G^{\prime}$ agree on all edges not contained in $U$, so $(G, q)$ and 
$(G, p)$ are equivalent. Since $(G, p)$ is globally rigid, $q$ and $p$ are congruent. Hence $\left(G^{\prime}, p\right)$ is globally rigid.

\subsection{The framework space}

Given a framwork $(G, p)$ and a vertex $v_{0}$ of $G$, its framework space $S_{G, p, v_{0}}$ consists of all $q \in \mathbb{R}^{2|V|}$ with $q\left(v_{0}\right)=(0,0)$ and $(G, q)$ equivalent to $(G, p)$. The following result on framework spaces is key to proving our main results. Its proof is similar to that of $[9$, Theorem 1.3].

Lemma 2.10. Suppose $(G, p)$ is a generic direction-length framework, e is a direction edge of $G, G$ is rigid, and $H=G-e$ is bounded and not rigid. Let $v_{0}$ be a vertex of $G$, let $p_{0}$ be obtained from $p$ by translating $v_{0}$ to the origin, and let $C$ be the connected component of the framework space $S_{H, p, v_{0}}$ containing $p_{0}$. Then $C$ is diffeomorphic to a circle. Furthermore, if $-p_{0} \notin C$ then $(G, p)$ is not globally rigid.

Proof. We may use [9, Lemma 1.2] and the hypotheses that $(G, p)$ is generic, $G$ is rigid and $H=G-e$ is not rigid, to deduce that $S_{H, p, v_{0}}$ is a smooth 1-dimensional manifold. The hypothesis that $H$ is bounded implies that $C$ is bounded. The fact that $C$ is closed now implies that $C$ is diffeomorphic to a circle.

Let $x, y$ be the end-vertices of $e$. If $q(x)=q(y)$ for some $q \in C$ then $(G, q)$ would be equivalent to $(G, p)$. This would contradict [7, Lemma 3.4], which tells us that $q$ should be injective when $(G, q)$ is equivalent to a rigid generic framework. Hence $q(x) \neq q(y)$ for all $q \in C$. Let $f: C \rightarrow \mathbb{S}^{1}$ be defined by $f(q)=(q(x)-q(y)) /\|q(x)-q(y)\|$. Then $f$ is a smooth map and we can use the fact that $p$ is generic to deduce that $p_{0}$ is a regular point of $f$ (we refer the reader to the proof of [9, Theorem 1.3] for more details).

To finish the proof we show that we can find $q \in C, q \neq p_{0}$ with $f(q)$ equal to $f\left(p_{0}\right)$ or $-f\left(p_{0}\right)$. Suppose that $-f\left(p_{0}\right) \notin f(C)$. Let $s: \mathbb{S}^{1} \backslash\left\{-f\left(p_{0}\right)\right\} \rightarrow \mathbb{R}$ be a diffeomorphism, e.g. stereographic projection. Then $s \circ f$ is a smooth map from $C$ to $\mathbb{R}$ and $p_{0}$ is a regular point of $s \circ f$. Thus we can find $q_{1}$ and $q_{2}$ in a neighbourhood of $p_{0}$ in $C$ with $(s \circ f)\left(q_{1}\right)<(s \circ f)\left(p_{0}\right)<(s \circ f)\left(q_{2}\right)$. Since there are two paths in $C$ joining $q_{1}$ and $q_{2}$, the intermediate value theorem implies we can find another realisation $q \in C, q \neq p_{0}$ with $(s \circ f)(q)=(s \circ f)\left(p_{0}\right)$, i.e. $f(q)=f\left(p_{0}\right)$.

We have shown that there is $q \in C, q \neq p_{0}$ with $f(q)$ equal to $f\left(p_{0}\right)$ or $-f\left(p_{0}\right)$. The hypothesis that $-p_{0} \notin C$ now implies that $q \neq-p_{0}$ and hence that $(G, q)$ is equivalent to but not congruent to $(G, p)$.

\subsection{Field extensions and genericity}

A mixed framework $(G, p)$ is quasi-generic if it is a translation of a generic framework. We will be mostly concerned with quasi-generic frameworks in standard position, i.e. with one vertex positioned at the origin. Such frameworks are characterised by the following elementary lemma. 
Lemma 2.11. [7] Let $(G, p)$ be a framework with vertices $\left\{v_{1}, v_{2}, \ldots, v_{n}\right\}, p\left(v_{1}\right)=$ $(0,0)$ and $p\left(v_{i}\right)=\left(p_{2 i-1}, p_{2 i}\right)$ for $2 \leq i \leq n$. Then $(G, p)$ is quasi-generic if and only if $\left\{p_{3}, p_{4}, \ldots, p_{2 n}\right\}$ is algebraically independent over $\mathbb{Q}$.

Given a vector $p \in \mathbb{R}^{d}, \mathbb{Q}(p)$ denotes the field extension of $\mathbb{Q}$ by the coordinates of $p$. We say that $p$ is generic in $\mathbb{R}^{d}$ if the coordinates of $p$ are algebraically independent over $\mathbb{Q}$. Given fields $K, L$ with $K \subseteq L$ the transcendence degree $t d[L: K]$ of $L$ over $K$ is the size of the largest subset of $L$ which is algebraically independent over $K$. A reformulation of Lemma 2.11 is that if $(G, p)$ is a framework with $n$ vertices, one of which is at the origin, then $(G, p)$ is quasi-generic if and only if $t d[\mathbb{Q}(p): \mathbb{Q}]=2 n-2$.

The following function plays an important role in rigidity theory. Let $(G, p)$ be a direction-length framework. For $v_{1}, v_{2} \in V$ with $p\left(v_{i}\right)=\left(x_{i}, y_{i}\right)$ let $l_{p}\left(v_{1}, v_{2}\right)=\left(x_{1}-x_{2}\right)^{2}+\left(y_{1}-y_{2}\right)^{2}$, and $s_{p}\left(v_{1}, v_{2}\right)=\left(y_{1}-y_{2}\right) /\left(x_{1}-x_{2}\right)$ whenever $x_{1} \neq x_{2}$. Suppose $e=v_{1} v_{2} \in D \cup L$. We say that $e$ is vertical in $(G, p)$ if $x_{1}=x_{2}$. The length of $e$ in $(G, p)$ is $l_{p}(e)=l_{p}\left(v_{1}, v_{2}\right)$, and the slope of $e$ is $s_{p}(e)=s_{p}\left(v_{1}, v_{2}\right)$, whenever $e$ is not vertical in $(G, p)$. Let $V=\left\{v_{1}, v_{2}, \ldots, v_{n}\right\}$ and $D \cup L=\left\{e_{1}, e_{2}, \ldots, e_{m}\right\}$. We view $p$ as a point $\left(p\left(v_{1}\right), p\left(v_{2}\right), \ldots, p\left(v_{n}\right)\right)$ in $\mathbb{R}^{2 n}$. Let $T$ be the set of all points $p \in \mathbb{R}^{2 n}$ such that $(G, p)$ has no vertical direction edges. Then the rigidity map $f_{G}: T \rightarrow \mathbb{R}^{m}$ is given by $f_{G}(p)=\left(h\left(e_{1}\right), h\left(e_{2}\right), \ldots, h\left(e_{m}\right)\right)$, where $h\left(e_{i}\right)=l_{p}\left(e_{i}\right)$ if $e_{i} \in L$ and $h\left(e_{i}\right)=s_{p}\left(e_{i}\right)$ if $e_{i} \in D$.

One can verify that each row in the Jacobian matrix of the rigidity map is a non-zero multiple of the corresponding row in the rigidity matrix, so these matrices have the same rank. Thus the rigidity matrix achieves its maximum rank at a framework $(G, p)$ when $p$ is a regular point of the rigidity map. Hence $p$ will be a regular point of $f$ whenever $(G, p)$ is generic.

Recall that $G=(V ; D, L)$ is independent if $D \cup L$ is independent in the (generic) rigidity matroid of $G$. The next result relates the genericity of $f_{G}(p)$ to the genericity of $p$ when $G$ is independent.

Lemma 2.12. [7] Suppose that $G$ is an independent mixed graph and $(G, p)$ is a quasi-generic realisation of $G$. Then $f_{G}(p)$ is generic.

We use $\bar{K}$ to denote the algebraic closure of a field $K$. Note that $t d[\bar{K}$ : $K]=0$. We say that $G$ is minimally rigid if it is rigid but $G-e$ is not rigid for any edge $e$; equivalently $G$ is both rigid and independent. The following lemma relates $\overline{\mathbb{Q}(p)}$ and $\overline{\mathbb{Q}\left(f_{G}(p)\right)}$ when $G$ is minimally rigid.

Lemma 2.13. [7] Let $G$ be a minimally rigid mixed graph and $(G, p)$ be a realisation of $G$ with no vertical direction edges and with $p(v)=(0,0)$ for some vertex $v$ of $G$. If $f_{G}(p)$ is generic then $\overline{\mathbb{Q}(p)}=\overline{\mathbb{Q}\left(f_{G}(p)\right)}$.

Lemmas 2.12 and 2.13 imply the following result for rigid mixed graphs.

Corollary 2.14. Let $G$ be a rigid mixed graph and $(G, p)$ be a quasi-generic realisation of $G$ with $p(v)=(0,0)$ for some vertex $v$ of $G$. Then $\overline{\mathbb{Q}(p)}=\overline{\mathbb{Q}\left(f_{G}(p)\right)}$. 
Proof. Let $H$ be a minimally rigid spanning subgraph of $G$. By Lemma 2.12, $f_{H}(p)$ is generic. Hence Lemma 2.13 gives $\overline{\mathbb{Q}(p)}=\overline{\mathbb{Q}\left(f_{H}(p)\right)}$. It is not difficult to see that $\overline{\mathbb{Q}\left(f_{H}(p)\right)} \subseteq \overline{\mathbb{Q}\left(f_{G}(p)\right)} \subseteq \overline{\mathbb{Q}(p)}$. Thus $\overline{\mathbb{Q}(p)}=\overline{\mathbb{Q}\left(f_{G}(p)\right)}$.

We also need the following lemma, which implies that every realisation of a rigid mixed graph which is equivalent to a generic realisation is quasi-generic.

Lemma 2.15. [7] Let $(G, p)$ be a quasi-generic realisation of a rigid mixed graph $G$. Suppose that $(G, q)$ is equivalent to $(G, p)$ and that $p(v)=(0,0)=q(v)$ for some vertex $v$ of $G$. Then $\overline{\mathbb{Q}(p)}=\overline{\mathbb{Q}(q)}$, so $(G, q)$ is quasi-generic.

\section{Realisations of graphs with given direction con- straints}

Here we give a result concerning the realisation of a graph as a direction-pure framework with given directions for its edges. We need the following concepts, introduced by Whiteley in [15]. A frame is a graph $G=(V, E)$ together with a map $q: E \rightarrow \mathbb{R}^{2}$. The incidence matrix of the frame $(G, q)$ is an $|E| \times 2|V|$ matrix $I(G, q)$ defined as follows. We first choose an arbitrary reference orientation for the edges of $E$. Each edge in $E$ corresponds to a row of $I(G, q)$ and each vertex of $V$ to two consecutive columns. The submatrix of $I(G, q)$ with row labeled by $e=u v \in E$ and pairs of columns labeled by $x \in V$ is $q(e)$ if $x=u$, is $-q(e)$ if

$x=v$, and is the 2-dimensional zero vector otherwise. It is known (see [15]) that when $q$ is generic, $I(G, q)$ is a linear representation of the matroid union of two copies of the cycle matroid of $G$. We may now use Theorem 2.5 to determine when $I(G, q)$ has linearly independent rows. For $X \subseteq V$, let $i_{G}(X)$ denote the number of edges of $G$ between vertices in $X$.

Theorem 3.1. Suppose $G=(V, E)$ is a graph and $q: E \rightarrow \mathbb{R}^{2}$ is generic. Then the rows of $I(G, q)$ are linearly independent if and only if $i_{G}(X) \leq 2|X|-2$ for all $\emptyset \neq X \subseteq V$.

We can use this result to show that a graph $G=(V, E)$ satisfying $i_{G}(X) \leq$ $2|X|-3$ for all $X \subseteq V$ with $|X| \geq 2$ can be realised as a direction-pure framework with a specified algebraically independent set of slopes for its edges, and that this realisation is unique up to translation and dilation when $|E|=2|V|-3$. Note that given any realisation of $G$, we can always translate a specified vertex $z_{0}$ to $(0,0)$ and dilate to arrange any specified distance $t$ between a specified pair of distinct vertices $x_{0}, y_{0}$. Our proof technique is similar to that developed in $[15]$.

Theorem 3.2. Let $G=(V, E)$ be a graph such that $i_{G}(X) \leq 2|X|-3$ for all $X \subseteq V$ with $|X| \geq 2$. Let $s$ be an injection from $E$ to $\mathbb{R}$ such that $\left\{s_{e}\right\}_{e \in E}$ is generic. Suppose $x_{0}, y_{0}, z_{0} \in V$ with $x_{0} \neq y_{0}$ and $t \neq 0$ is a real number. Then there exists an injection $p: V \rightarrow \mathbb{R}^{2}$ such that $\left\|p\left(x_{0}\right)-p\left(y_{0}\right)\right\|=t, p\left(z_{0}\right)=(0,0)$ and, for all $e=u v \in E, p(u)-p(v) \in\left\langle\left(1, s_{e}\right)\right\rangle$. Furthermore, if $|E|=2|V|-3$, then $p$ is unique up to dilation by -1 through $(0,0)$. 
Proof. We will construct $p$ as a combination of vectors in the nullspaces of certain frames. First consider a generic frame $q$ on $G$ such that $q(e)$ is a scalar multiple of $\left(-s_{e}, 1\right)$ for every $e \in E$. Then for any $p$ in the nullspace of $I(G, q)$ and $e=u v \in E$ we have $p(u)-p(v) \in\left\langle\left(1, s_{e}\right)\right\rangle$. However, $p$ need not be injective. To address this issue, we instead choose a pair of vertices $x, y \in V$, and consider the graph $H$ obtained by adding the edge $f=x y$ to $G$ (which may be parallel to an existing edge). Now let $\left(H, q^{\prime}\right)$ be a generic frame such that $\left.q^{\prime}\right|_{E}=q$. For all $X \subseteq V$ with $|X| \geq 2$, we have $i_{H}(X) \leq i_{G}(X)+1 \leq 2|X|-2$ by hypothesis. Theorem 3.1 now implies that the incidence matrix $I\left(H, q^{\prime}\right)$ has linearly independent rows. Thus $\operatorname{rank} I\left(H, q^{\prime}\right)=\operatorname{rank} I(G, q)+1$. Writing $Z_{H}$ for the null space of $I\left(H, q^{\prime}\right)$ and $Z_{G}$ for the null space of $I(G, q)$, we have $\operatorname{dim} Z_{G}=\operatorname{dim} Z_{H}+1$, so we can choose $p_{f} \in Z_{G} \backslash Z_{H}$. Then we necessarily have $p_{f}(x) \neq p_{f}(y)$. Taking a suitable linear combination of the vectors $p_{f}$, for all possible new edges $f=x y, x, y \in V$, we may construct a vector $p$ in $Z_{G}$ with $p(x) \neq p(y)$ for all $x, y \in V$. Since $p_{f}(u)-p_{f}(v) \in\left\langle\left(1, s_{e}\right)\right\rangle$ for each $f$ we also have $p(u)-p(v) \in\left\langle\left(1, s_{e}\right)\right\rangle$. Furthermore, as noted before the proof, we can translate and dilate to satisfy the other conditions, thus constructing the required map $p$.

We next show uniqueness when $|E|=2|V|-3$. We have $\operatorname{dim} Z_{G}=2|V|-$ $\operatorname{rank} I(G, q)=2|V|-|E|=3$. Define $p_{1}, p_{2}: V \rightarrow \mathbb{R}^{2}$ by $p_{1}(v)=(1,0)$ and $p_{2}(v)=(0,1)$ for all $v \in V$. Note that $p_{1}, p_{2} \in Z_{G}$. Also, $p, p_{1}, p_{2}$ are linearly independent, since $p\left(z_{0}\right)=(0,0), p_{1}\left(z_{0}\right)=(1,0)$ and $p_{2}\left(z_{0}\right)=(0,1)$, so $\left\{p, p_{1}, p_{2}\right\}$ is a basis for $Z_{G}$. Now suppose that $p^{\prime}: V \rightarrow \mathbb{R}^{2}$ has the properties described in the first part of the lemma. Then $p^{\prime} \in Z_{G}$ so $p^{\prime}=a p+b p_{1}+c p_{2}$ for some $a, b, c \in \mathbb{R}$. Since $p^{\prime}\left(z_{0}\right)=p\left(z_{0}\right)=(0,0)$ we have $b=c=0$. Since $\left\|p^{\prime}\left(x_{0}\right)-p^{\prime}\left(y_{0}\right)\right\|=t=\left\|p\left(x_{0}\right)-p\left(y_{0}\right)\right\|$ we have $p^{\prime} \in\{p,-p\}$.

\section{Direction reduction}

In this section we will derive Theorem 1.4. We also prove a lemma which characterises when a rigid direction-independent mixed graph is direction reducible. We first consider the reduction operation $R_{1}$.

Lemma 4.1. Suppose $(G, p)$ is a generic realisation of a mixed graph $G=$ $(V ; D, L)$ and that $e=u v \in D$ belongs to a direction-pure $M$-circuit $H=$ $(U ; F, \emptyset)$ of $G$. Then $(G, p)$ is globally rigid if and only if $(G-e, p)$ is globally rigid.

Proof. If $(G-e, p)$ is globally rigid then $(G, p)$ is clearly globally rigid. Conversely, suppose that $(G, p)$ is globally rigid and $(G-e, q)$ is equivalent to $(G-e, p)$. Since $H$ is a direction-pure circuit, $\left(H-e,\left.p\right|_{U}\right)$ is direction-rigid. Hence $\left(H-e,\left.p\right|_{U}\right)$ is globally direction-rigid by Lemma 1.1. Thus $q(u)-q(v)$ is a scalar multiple of $p(u)-p(v)$, and hence $(G, q)$ is equivalent to $(G, p)$. Since $G$ is globally rigid, $q$ is congruent to $p$. This shows that $(G-e, p)$ is globally rigid.

We next consider the reduction operation $R_{2}$. 
Lemma 4.2. Let $(G, p)$ be a quasi-generic realisation of a rigid mixed graph $G=(V ; D, L)$. Suppose that $G$ has a proper induced subgraph $H=(U ; F, L)$ such that the graph $G / H$ obtained by contracting $H$ to a single vertex (deleting all edges contained in $H$ and keeping all other edges, possibly as parallel edges) has only direction edges and is the union of two edge-disjoint spanning trees. Then $(G, p)$ is globally rigid if and only if $\left(H,\left.p\right|_{H}\right)$ is globally rigid.

Proof. First suppose that $\left(H,\left.p\right|_{H}\right)$ is globally rigid. Let $G^{\prime}$ be constructed from $G$ by substituting $H$ by a minimally rigid graph $H^{\prime}=\left(U ; F^{\prime},\left\{l^{\prime}\right\}\right)$ with exactly one length edge $l^{\prime}$. Then $G^{\prime}$ is rigid by Lemma 2.8. Since $G^{\prime}$ is rigid and has exactly one length edge, $\left(G^{\prime}, p\right)$ is globally rigid by Lemma 1.2 . Thus $(G, p)$ is globally rigid by Lemma 2.9 .

Conversely, suppose that $\left(H,\left.p\right|_{H}\right)$ is not globally rigid. Then there exists an equivalent but non-congruent framework $(H, \tilde{q})$. Without loss of generality we may suppose that $p(u)=(0,0)=\tilde{q}(u)$ for some $u \in V(H)$. We will construct a framework $(G, q)$ which is equivalent to $(G, p)$ and has $\left.q\right|_{H}=\tilde{q}$. Let $D^{*}=D \backslash F$ be the set of edges of $G / H$ and $m$ be the number of vertices of $G / H$. Then $\left|D^{*}\right|=2 m-2$, as $G / H$ is the union of two edge-disjoint spanning trees. Since $G$ is rigid we have

$$
2|V|-2=r(G) \leq\left|D^{*}\right|+r(H) \leq 2 m-2+2|V(H)|-2=2|V|-2 .
$$

Thus equality must hold throughout. In particular, $r(H)=2 \mid V(H)) \mid-2$, so $H$ is rigid.

We again consider the rigid mixed graph $G^{\prime}=\left(V ; D^{\prime},\left\{l^{\prime}\right\}\right)$ with exactly one length edge defined in the first paragraph of the proof. Since $G^{\prime}$ has $\left|D^{*}\right|+$ $2|V(H)|-2=2|V|-2$ edges, it is minimally rigid. $\mathrm{t}$

Define $s: D^{\prime} \cup L^{\prime} \rightarrow \mathbb{R}$ by $s(e)=s_{\tilde{q}}(e)$ for $e \in F^{\prime}, s\left(l^{\prime}\right)=l_{\tilde{q}}\left(l^{\prime}\right)$, and $s(e)=$ $s_{p}(e)$ for $e \in D^{*}$. We will use Theorem 3.2 to construct a framework $\left(G^{\prime}, q\right)$ such that $s_{q}(e)=s(e)$ for all edges $e$ of $G^{\prime}$. To do this, we first need to show that $\left.s\right|_{D^{\prime}}$ is generic. We will prove the stronger result that $s$ is generic by showing that $t d[\overline{\mathbb{Q}(s)}: \mathbb{Q}]=\left|D^{\prime}\right|+\left|L^{\prime}\right|=2|V|-2$. We have $t d[\overline{\mathbb{Q}(p)}: \mathbb{Q}]=2|V|-2$, as $p$ is quasi-generic and $p(u)=(0,0)$, so it suffices to prove that $\overline{\mathbb{Q}(s)}=\overline{\mathbb{Q}(p)}$. Since $G$ is rigid, Corollary 2.14 gives $\overline{\mathbb{Q}\left(f_{G}(p)\right)}=\overline{\mathbb{Q}(p)}$. Also, $s$ is obtained from $f_{G}(p)$ by replacing the values $f_{H}\left(\left.p\right|_{U}\right)$ by the values $f_{H^{\prime}}(\tilde{q})$, so we need to show that these generate the same algebraic closure over $\mathbb{Q}$. Since $(H, \tilde{q})$ is equivalent to $\left(H,\left.p\right|_{U}\right)$, Lemma 2.15 gives $\overline{\mathbb{Q}(\tilde{q})}=\overline{\mathbb{Q}\left(\left.p\right|_{U}\right)}$. Since $\left.p\right|_{U}$ is quasi-generic, it follows that $\tilde{q}$ is quasi-generic. Then, since $H$ and $H^{\prime}$ are rigid, two applications of Corollary 2.14, give $\overline{\mathbb{Q}\left(f_{H}\left(\left.p\right|_{U}\right)\right)}=\overline{\mathbb{Q}\left(\left.p\right|_{U}\right)}$ and $\overline{\mathbb{Q}\left(f_{H^{\prime}}(\tilde{q})\right)}=\overline{\mathbb{Q}(\tilde{q})}$. Putting these three equalities together gives

$$
\overline{\mathbb{Q}\left(f_{H^{\prime}}(\tilde{q})\right)}=\overline{\mathbb{Q}(\tilde{q})}=\overline{\mathbb{Q}\left(\left.p\right|_{U}\right)}=\overline{\mathbb{Q}\left(f_{H}\left(\left.p\right|_{U}\right)\right)},
$$

which is what we needed to prove $\overline{\mathbb{Q}(s)}=\overline{\mathbb{Q}(p)}$. Therefore $s$ is generic. Now we can apply Theorem 3.2 , with $x_{0} y_{0}$ equal to the unique length edge of $G^{\prime}$, to obtain a realisation $\left(G^{\prime}, q\right)$ with $f_{G^{\prime}}(q)=s$. By construction, $\left(H^{\prime},\left.q\right|_{U}\right)$ is equivalent to $\left(H^{\prime}, \tilde{q}\right)$. But $H^{\prime}$ is globally rigid by Lemma 1.2 , so $\left.q\right|_{U}$ is congruent 
to $\tilde{q}$. Hence we can apply a translation, and possibly a dilation by -1 , to obtain $\left.q\right|_{U}=\tilde{q}$.

Since $(H, \tilde{q})$ is equivalent to $\left(H,\left.p\right|_{H}\right)$ and $s_{q}(e)=s(e)=s_{p}(e)$ for all $e \in$ $D^{*},(G, q)$ is equivalent to $(G, p)$ and satisfies $\left.q\right|_{U}=\tilde{q}$. Since $(H, \tilde{q})$ is not congruent to $\left(H,\left.p\right|_{U}\right),(G, q)$ is not congruent to $(G, p)$. Thus $(G, p)$ is not globally rigid.

Theorem 1.4 follows immediately from Lemmas 4.1 and 4.2 . We close this section with a result which characterises when a rigid, direction-independent mixed graph $G$ is direction reducible to a given subgraph $H$. This lemma will be used in Section 7 to prove Theorem 1.6.

Lemma 4.3. Let $G=(V ; D, L)$ be a rigid mixed graph and $H=\left(V^{\prime} ; D^{\prime}, L^{\prime}\right)$ be an induced proper subgraph of $G$. Suppose that no edge of $D \backslash D^{\prime}$ is contained in an $M$-circuit of $G$. Then $G$ is direction reducible to $H$ if and only if $L^{\prime}=L$ and $\left|D \backslash D^{\prime}\right|=2\left|V \backslash V^{\prime}\right|$.

Proof. Since no edge of $D \backslash D^{\prime}$ is contained in an $M$-circuit of $G, G$ is direction reducible to $H$ if and only if $L^{\prime}=L$ and the graph $F=\left(V^{\prime \prime} ; D^{\prime \prime}, \emptyset\right)$ obtained by contracting $H$ to a single vertex $v_{H}$ is the union of two edge-disjoint spanning trees. Thus, if $G$ is direction reducible to $H$, then we have $L^{\prime}=L$ and $\left|D \backslash D^{\prime}\right|=$ $\left|D^{\prime \prime}\right|=2\left|V^{\prime \prime}\right|-2=2\left|V \backslash V^{\prime}\right|$.

We next assume that $L^{\prime}=L$ and $\left|D \backslash D^{\prime}\right|=2\left|V \backslash V^{\prime}\right|$ and show that $F$ is the union of two edge-disjoint spanning trees. Suppose not. Then by Theorem 2.5, there exists $X \subseteq V^{\prime \prime}$ with $|X| \geq 2$ and $i_{F}(X) \geq 2|X|-1$. If $v_{H} \notin X$ then the fact that no edge of $D \backslash D^{\prime}$ is contained in an $M$-circuit of $G$ implies that $i_{F}(X)=i_{G}(X) \leq 2|X|-3$, a contradiction. Thus $v_{H} \in X$. Since $\left|D^{\prime \prime}\right|=$ $\left|D \backslash D^{\prime}\right|=2\left|V^{\prime \prime}\right|-2$ there are at most $\left(2\left|V^{\prime \prime}\right|-2\right)-(2|X|-1)=2\left|V^{\prime \prime} \backslash X\right|-1$ edges in $F$ which are not induced by $X$. It follows that

$r(G) \leq r\left(G\left[V \backslash\left(V^{\prime \prime} \backslash X\right)\right]\right)+\left(2\left|V^{\prime \prime} \backslash X\right|-1\right) \leq\left(2\left|V \backslash\left(V^{\prime \prime} \backslash X\right)\right|-2\right)+\left(2\left|V^{\prime \prime} \backslash X\right|-1\right)$

which gives

$$
r(G) \leq 2|V|-3 .
$$

This contradicts the hypothesis that $G$ is rigid. Thus $F$ is the union of two edge-disjoint spanning trees and $G$ is direction reducible to $H$.

\section{$5 \quad$ Direction irreducible mixed graphs}

Theorem 1.4 enables us to reduce the problem of characterising globally rigid generic direction-length frameworks to the case when the underlying graph is direction irreducible. In this section we prove a structural lemma for direction irreducible mixed graphs which have a globally rigid generic realisation even though they are not redundantly rigid. This will be used in the next section to construct two equivalent but non-congruent generic realisations of a mixed graph which is direction irreducible but not redundantly rigid. 
Lemma 5.1. Let $G=(V ; D, L)$ be a direction irreducible mixed graph which has $|L| \geq 2$ and is not redundantly rigid. Suppose that $(G, p)$ is a globally rigid generic realisation of $G$. Then

(a) $G-e$ is bounded for all $e \in D$,

(b) $r(G-e)=r(G)-1$ for all $e \in D$, and

(c) every length edge of $G$ belongs to a length-pure $M$-circuit of $G$.

Proof. (a) First note that $G$ is direction-independent, since $G$ is direction irreducible. Now suppose for a contradiction that $G-e$ is not bounded for some $e \in D$. We will show that $G$ has a direction reduction. Let $H_{1}, H_{2}, \ldots, H_{m}$ be the bounded components of $G-e$. Then each length edge of $G$ is contained in one of the subgraphs $H_{i}$. Let $D^{*} \subseteq D$ be the set of all edges of $G$ joining distinct subgraphs $H_{i}$, and $H$ be the graph obtained from $G$ by contracting each $H_{i}$ to a single vertex. Since $G$ is rigid, $G$ is bounded. Since $G$ is direction-independent, Lemma 2.4 implies that the graph $G / L$ obtained from $G$ by contracting each length edge has two edge-disjoint spanning trees. Since $H$ can be obtained from $G / L$ by contracting a (possibly empty) set of direction edges, $H$ also has two edge-disjoint spanning trees. In particular, $\left|D^{*}\right| \geq 2 m-2$. On the other hand, Lemma 2.6 implies that $\left|D^{*}-e\right| \leq 2 m-3$. Thus $e \in D^{*},\left|D^{*}\right|=2 m-2$, and $H$ is the union of two edge-disjoint spanning trees. Since $G$ is rigid we have

$$
2|V|-2=r(G) \leq\left|D^{*}\right|+\sum_{i=1}^{m} r\left(H_{i}\right) \leq 2 m-2+\sum_{i=1}^{m}\left(2\left|V\left(H_{i}\right)\right|-2\right)=2|V|-2 .
$$

Thus equality must hold throughout. In particular, $r\left(H_{i}\right)=2\left|V\left(H_{i}\right)\right|-2$ for each $i$, so each subgraph $H_{i}$ is rigid.

Let $G^{\prime}=\left(V ; D^{\prime}, L^{\prime}\right)$ be obtained from $G$ by substituting each non-trivial subgraph $H_{i}$ by a minimally rigid graph $H_{i}^{\prime}$ with exactly one length edge. Each framework $\left(H_{i}^{\prime},\left.p\right|_{H_{i}^{\prime}}\right)$ is globally rigid by Lemma 1.2. Thus repeated applications of Lemma 2.9 imply that $\left(G^{\prime}, p\right)$ is globally rigid. On the other hand, $\left|D^{\prime}\right|+\left|L^{\prime}\right|=$ $\left|D^{*}\right|+\sum_{i=1}^{m} r\left(H_{i}\right)=2|V|-2$, so $G^{\prime}$ is minimally rigid. Theorem 2.7(a) now implies that $G^{\prime}$ has exactly one length edge. Since $H_{i}^{\prime}$ contains a length edge whenever $H_{i}$ is non-trivial, $G-e$ has exactly one non-trivial bounded component, $H_{1}$ say. Since $G / H_{1}=H$ and $H$ is the union of two edge-disjoint spanning trees, $G$ is direction reducible to $H_{1}$. This contradicts the hypothesis that $G$ is direction irreducible.

(b) Suppose that $r(G-e)=r(G)$ for some $e \in D$. Then $e$ is contained in an $M$-circuit $C$ of $G$. Since $G$ is direction-independent, $C$ must be a mixed $M$ circuit. Since $G$ is not redundantly rigid, $G-f$ is not rigid for some $f \in D \cup L$. Theorem 2.7(a) implies that $f \in D$. Clearly $f$ is not an edge of $C$ and hence $C$ is a non-trivial rigid subgraph of $G-e$. Theorem 2.7(b) now implies that $G-f$ is unbounded, contradicting (a).

(c) Choose $e \in L$. Then $e$ belongs to an $M$-circuit $C^{\prime}$ of $G$ by Theorem 2.7(a). By (b), $C^{\prime}$ cannot be a mixed $M$-circuit. Hence $C^{\prime}$ is length-pure. 


\section{Proof of Theorem 1.5}

Every generic realisation of a direction irreducible, 2-connected, direction-balanced, redundantly rigid graph is globally rigid by Theorem 1.3 and Lemma 2.2. So it only remains to show necessity in Theorem 1.5 . Hence we may suppose that $G=(V ; D, L)$ is a direction irreducible, mixed graph with $|L| \geq 2$, and that every generic realisation of $G$ is globally rigid. The fact that some generic realisation of $G$ is generically globally rigid implies that $G$ is 2-connected and direction-balanced, see [6]. We complete the proof by applying Theorem 6.1 below to deduce that $G$ must also be redundantly rigid. The proof idea is to show that if $G$ is not redundantly rigid, then for any given generic realisation $(G, p)$, we can construct a sequence of generic realisations $q_{0}, q_{1}, \ldots, q_{t}$ such that $t \leq|D|$ and $\left(G, q_{t}\right)$ is not globally rigid. We construct this sequence from $(G, p)$ by first reflecting $(G, p)$ in the $x$-axis to obtain $\left(G, q_{0}\right)$, and then recursively "correcting" the changed direction constraints back to their original values in $(G, p)$. Every time we "correct" a direction constraint, we obtain a new realisation in our sequence.

Theorem 6.1. Let $G=(V ; D, L)$ be a direction irreducible mixed graph with $|L| \geq 2$ such that $G$ is not redundantly rigid. Then some generic realisation of $G$ is not globally rigid.

Proof. We proceed by contradiction. Assume that all generic realisations of $G$ are globally rigid. By Lemma 5.1(b) and (c), every length edge of $G$ is contained in a length-pure $M$-circuit of $G$, and no direction edge of $G$ is contained in any $M$-circuit of $G$. Let $D=\left\{d_{0}, d_{1}, \ldots, d_{k}\right\}$, let $G_{1}=\left(V_{1} ; \emptyset, L_{1}\right)$ be a non-trivial $M$-component of $G$ and let $v_{0} \in V_{1}$.

Let $(G, p)$ be a quasi-generic realisation of $G$ with $p\left(v_{0}\right)=(0,0)$ and let $\left(G, q_{0}\right)$ be the quasi-generic realisation obtained by reflecting $(G, p)$ in the $x$ axis. Then $(G-D, p)$ is equivalent to $\left(G-D, q_{0}\right)$. In addition we have $s_{q_{0}}\left(d_{i}\right)=$ $-s_{p}\left(d_{i}\right)$ for all $d_{i} \in D$, so $(G, p)$ and $\left(G, q_{0}\right)$ are not equivalent.

Claim 6.2. For all $j \in\{0,1, \ldots, k+1\}$ there exists a quasi-generic framework $\left(G, q_{j}\right)$ with $q_{j}\left(v_{0}\right)=(0,0)$, rigidity map $f_{G}\left(q_{j}\right)=\left(h_{q_{j}}(e)\right)_{e \in E}$ given by

$$
h_{q_{j}}(e)=\left\{\begin{array}{l}
s_{q_{0}}(e) \text { when } e \in\left\{d_{j}, d_{j+1}, \ldots, d_{k}\right\} \\
h_{p}(e) \quad \text { otherwise, }
\end{array}\right.
$$

and with the property that that $\left(G_{1},\left.q_{j}\right|_{V_{1}}\right)$ can be obtained from $\left(G_{1},\left.q_{0}\right|_{V_{1}}\right)$ by a rotation about the origin.

Proof. We proceed by induction on $j$. If $j=0$ then the claim holds trivially for $\left(G, q_{0}\right)$. Hence suppose that the required framework $\left(G, q_{j}\right)$ exists for some $0 \leq j<k+1$. The quasi-generic framework $\left(G-d_{j}, q_{j}\right)$ is bounded but not rigid by Lemma 5.1(a) and (b) (since boundedness and rigidity are generic properties). Since $\left(G, q_{j}\right)$ is globally rigid by assumption, Lemma 2.10 implies that we can continuously move $\left(G-d_{j}, q_{j}\right)$ to form $\left(G-d_{j},-q_{j}\right)$ whilst keeping $v_{0}$ 
fixed at the origin and maintaining all edge constraints. During this motion, the direction of the missing edge $d_{j}=u_{j} v_{j}$ changes continuously from $q_{j}\left(v_{j}\right)-q_{j}\left(u_{j}\right)$ to $-\left(q_{j}\left(v_{j}\right)-q_{j}\left(u_{j}\right)\right)$, a rotation by $180^{\circ}$. So at some point in this motion we must pass through a realisation $\left(G-d_{j}, q_{j+1}\right)$ at which the slope of this missing edge is $s_{p}\left(d_{j}\right)$. We can now add the edge $d_{j}$ back to this realisation to obtain the desired framework $\left(G, q_{j+1}\right)$. Note that since $G_{1}$ is $M$-connected, it is a length-rigid subgraph of $G-d_{j}$. Since the motion of $\left(G-d_{j}, q_{j}\right)$ is continuous and keeps $v_{0}$ fixed at the origin, this implies that $\left(G_{1},\left.q_{j+1}\right|_{V_{1}}\right)$ can be obtained from $\left(G_{1},\left.q_{j}\right|_{V_{1}}\right)$ by a rotation about the origin.

It remains to show that $\left(G, q_{j+1}\right)$ is quasi-generic. Let $H$ be a minimally rigid spanning subgraph of $G$. Since $h_{q_{j+1}}(e)= \pm h_{p}(e)$ for all $e \in E(G)$ we have $\mathbb{Q}\left(f_{H}\left(q_{j+1}\right)\right)=\mathbb{Q}\left(f_{H}(p)\right)$. Since $f_{H}(p)$ is generic by Lemma 2.12 , Lemma 2.13 implies that

$$
\operatorname{td}\left[\overline{\mathbb{Q}\left(q_{j+1}\right)}: \mathbb{Q}\right]=\operatorname{td}\left[\overline{\mathbb{Q}\left(f_{H}\left(q_{j+1}\right)\right)}: \mathbb{Q}\right]=\operatorname{td}\left[\overline{\mathbb{Q}\left(f_{H}(p)\right)}: \mathbb{Q}\right]=2|V|-2 .
$$

We can now use Lemma 2.11 to deduce that $\left(H, q_{j+1}\right)$, and hence also $\left(G, q_{j+1}\right)$, are quasi-generic.

Applying Claim 6.2 with $j=k+1$, we obtain a quasi-generic realisation $q_{k+1}$ of $G$ which is equivalent to $(G, p)$, has $q_{k+1}\left(v_{0}\right)=(0,0)$, and is such that $\left(G_{1},\left.q_{k+1}\right|_{V_{1}}\right)$ can be obtained from $\left(G_{1},\left.q_{0}\right|_{V_{1}}\right)$ by a rotation about the origin. Since $q_{0}$ was obtained from $p$ by reflecting $G$ across the $x$-axis, we have

$$
q_{k+1}(v)=R Z p(v) \text { for all } v \in V_{1}
$$

where $R$ and $Z$ are the $2 \times 2$ matrices representing this rotation and reflection. Since $\left(G_{1},\left.p\right|_{V_{1}}\right)$ is a quasi-generic framework with at least four vertices and $R Z$ acts on $\mathbb{R}^{2}$ as a reflection in some line through the origin, we have $q_{k+1}(v) \neq$ $\pm p(v)$ for some $v \in V_{1}$. Hence $\left.q_{k+1}\right|_{V_{1}}$ is not congruent to $\left.p\right|_{V_{1}}$, and $q_{k+1}$ is not congruent to $p$. This implies that $(G, p)$ is not globally rigid and contradicts our initial assumption that all generic realisations of $G$ are globally rigid.

Theorem 6.1 and the preceding discussion immediately imply Theorem 1.5.

\section{Proof of Theorem 1.6}

Lemma 2.2 implies that we can replace redundant rigidity with $M$-connectivity in the statement of Theorem 1.5. Since 2-connectivity is a property of $M$ connected graphs (see [2]), we can then remove this condition. This gives us the equivalent statement:

Theorem 7.1. Let $G=(V ; D, L)$ be a direction irreducible mixed graph with $|L| \geq 2$. Then all generic realisations of $G$ are globally rigid if and only if $G$ is direction-balanced and $M$-connected. 
With this statement, we can finally prove Theorem 1.6.

Proof of Theorem 1.6. We first prove necessity. Suppose all generic realisations of $G=(V ; D, L)$ are globally rigid. Then $G$ is rigid. If $|L|=1$, we are done. So suppose $|L| \geq 2$. Choose a minimal subgraph $H$ of $G$ such that $H$ can be obtained by a direction reduction of $G$ (we allow the possibility that $H=G$.) Then $H$ is direction irreducible and and $L$ is contained in $H$. Theorem 1.4 implies that all generic realisations of $H$ are globally rigid. Theorem 7.1 now tells us that $H$ is the required direction-balanced, $M$-connected mixed subgraph of $G$.

We next prove sufficiency. Suppose that $G$ is rigid and let $(G, p)$ be a generic realisation of $G$. If $|L|=1$, then $(G, p)$ is globally rigid by Lemma 1.2. So suppose that $|L| \geq 2$, and that $G$ has a direction-balanced, $M$-connected mixed subgraph $H$ containing all edges in $L$. We will show that $(G, p)$ is globally rigid by induction on $|E(G)|$. If $G=H$ then $(G, p)$ is globally rigid by Theorem 1.3. Hence we may assume that $G \neq H$. If $G-e$ is rigid for some edge $e \in E(G) \backslash E(H)$ then we may apply induction to $G-e$ to deduce that $(G-e, p)$ is globally rigid. Hence we may assume that no edge of $E(G) \backslash E(H)$ belongs to an $M$-circuit of $G$. This, and the fact that $G$ and $H$ are both rigid, gives

$$
|E(G)|-|E(H)|=\mathrm{r}(G)-\mathrm{r}(H)=2|V(G)|-2|V(H)| .
$$

Lemma 4.3 now implies that $G$ is direction reducible to $H$. By induction, $\left(H,\left.p\right|_{H}\right)$ is globally rigid. Theorem 1.4 now tells us that $(G, p)$ is globally rigid.

\section{Algorithmic considerations}

It is not difficult to see that a mixed graph $G=(V ; D, L)$ has an $M$-connected, direction balanced, mixed subgraph which contains $L$ if and only if some $M$ connected component of $G$ is direction balanced, mixed and contains $L$. Theorem 1.6 now reduces the problem of checking whether every generic realisation of $G$ is globally rigid to that of determining the $M$-connected components of $G$ and checking whether one of them contains $L$ and is direction balanced.

There exist efficient algorithms to check whether a mixed graph $G=(V ; D, L)$ satisfies the sparsity condition of Theorem 2.1. For any $F \subseteq E$, the condition $F^{\prime} \leq 2\left|V\left(F^{\prime}\right)\right|-2$ for all $\emptyset \neq F^{\prime} \subseteq F$ holds if and only if $F$ can be covered by two forests, which can be tested in $O\left(n^{3 / 2} \log n^{2} / m\right)$ time [3], where $n$ and $m$ denote the number of vertices and edges, respectively. For $F \subseteq E$ or $F \subseteq D$, the condition $F^{\prime} \leq 2\left|V\left(F^{\prime}\right)\right|-3$ for all $\emptyset \neq F^{\prime} \subseteq F$ is equivalent to independence in the well-known bar-joint rigidity matroid and can be tested in $O\left(n^{2}\right)$ time, see $[1,11]$. By using these algorithms one can test independence in the directionlength rigidity matroid. This allows us to check whether $G$ is rigid and find its $M$-connected components. We again refer to $[1,11]$ for more details.

Testing whether $G$ is direction balanced can be done in linear time. This follows by observing that $G$ is direction balanced if and only if all 2-separations 
$\left(H_{1}, H_{2}\right)$ of $G$, in which $H_{2}$ is minimal, are direction balanced. It is straightforward to obtain these special 2-separations from the cleavage units (i.e. 3connected components) of $G$, which can be listed in $O(n+m)$ time [4].

\section{Closing remarks}

The question of deciding whether direction-length global rigidity is a generic property remains open. Theorem 1.3 shows that it is a generic property when the underlying graph is $M$-connected, and the necessary conditions for global rigidity given in [6] show that it is also a generic property if the underlying graph is not 2-connected or is not direction-balanced. Theorem 1.4 and Lemma 2.2 reduce the question to the case when the underlying graph is direction irreducible and is not redundantly rigid. Theorem 1.5 tells us that a direction irreducible mixed graph $G$ which is not redundantly rigid has a generic realisation which is not globally rigid, but it is still conceivable that $G$ may also have a generic realisation which is globally rigid. We believe that this is not the case:

Conjecture 9.1. Suppose $(G, p)$ is a generic realisation of a direction irreducible mixed graph $G$ with at least two length edges. Then $(G, p)$ is globally rigid if and only if $G$ is 2-connected, direction-balanced, and redundantly rigid.

If true, Conjecture 9.1 would imply that a generic direction-length framework is globally rigid if and only if it satisfies the conditions in Theorem 1.6:

Conjecture 9.2. A generic direction-length framework $(G, p)$ is globally rigid if and only if $G=(V ; D, L)$ is rigid, and either $|L|=1$ or $G$ has a directionbalanced, $M$-connected mixed subgraph which contains all edges in $L$.

In these final pages, we show that Conjecture 9.1, and hence also Conjecture 9.2 , hold in the special case when the length edges of $G$ induce a length-rigid subgraph. As noted above, Theorem 1.3, Lemma 2.2 and [6] imply it only remains to establish the necessity of the condition that $G$ is redundantly rigid. We will prove this in Theorem 9.4, but first we need the following rather technical lemma.

Lemma 9.3. Let $G=(V ; D, L)$ be a rigid mixed graph, $H=(U ; \emptyset, L)$ be the length-pure subgraph induced by $L$, and $u \in U$. Suppose that $H$ is length rigid, $r(G-e)=r(G)-1$ for all $e \in D$, and $G-e_{0}$ is bounded for some $e_{0} \in D$. Let $(G, p)$ be a quasi-generic framework with $p(u)=(0,0)$ and $C$ be the connected component of the configuration space $S_{G-e_{0}, p, u}$ which contains $p$. Then $-p \in C$.

Proof. The idea is to rotate $\left(H,\left.p\right|_{U}\right)$ by $\theta$ radians about $p(u)=(0,0)$ and use Theorem 3.2 to show that, for almost all values of $\theta$, we can extend the resulting framework $\left(H, q_{\theta}\right)$ to a framework $\left(G-e_{0}, p_{\theta}\right)$ which is equivalent to $\left(G-e_{0}, p\right)$. To apply Theorem 3.2, we construct $G^{\prime}$ from $G$ by substituting a minimally rigid graph $H^{\prime}$ with exactly one length edge for $H$ and then show that the required set of edge slopes for $\left(G^{\prime}-e_{0}, p_{\theta}\right)$ is algebraically independent over $\mathbb{Q}$. 
Let $H^{\prime}=\left(U ; D^{\prime}, L^{\prime}\right)$ be a minimally rigid graph on the same vertex set as $H$ with exactly one length edge and let $G^{\prime}$ be obtained from $G$ by replacing $H$ by $H^{\prime}$. We first show that $G^{\prime}-e_{0}$ is minimally rigid. Since $G$ is rigid, $H$ is lengthrigid and $r(G-e)=r(G)-1$ for all $e \in D$, we have $|D|=2|V|-2-(2|U|-3)$ and hence $\left|D-e_{0}\right|=2|V|-2|U|$. Since $H^{\prime}$ has $2|U|-2$ edges, this implies that $G^{\prime}$ has $2|V|-2$ edges. It remains to show that $G^{\prime}-e_{0}$ is rigid. Since $G-e_{0}$ is bounded, $\left(G-e_{0}\right)^{+}$is rigid by Lemma 2.3. Since $G^{\prime}-e_{0}$ can be obtained from $\left(G-e_{0}\right)^{+}$by substituting $H^{+}$with $H^{\prime}$, it is rigid by Lemma 2.8. Therefore $G^{\prime}-e_{0}$ is minimally rigid.

For each $\theta \in[0,2 \pi)$ let $q_{\theta}: U \rightarrow \mathbb{R}^{2}$ be the configuration obtained by an anticlockwise rotation of $\left.p\right|_{U}$ through $\theta$ radians about $(0,0)$. Write $B=$ $\left\{q_{\theta}: \theta \in[0,2 \pi)\right\}$, and let $B^{*}$ be the set of all configurations $q_{\theta} \in B$ such that the set of slopes $\left\{s_{p}(e)\right\}_{e \in D-e_{0}} \cup\left\{s_{q_{\theta}}(e)\right\}_{e \in D^{\prime}}$ is defined and is algebraically independent over $\mathbb{Q}$. We claim that $B^{*}$ is a dense subset of $B$. First we note that $q_{0}=\left.p\right|_{U} \in B^{*}$, as $G^{\prime}-e_{0}$ is independent, so Lemma 2.12 implies that $f_{G^{\prime}}(p)$ is generic. To see the effect of a rotation by $\theta$, consider an edge $e=v_{1} v_{2}$ in $D^{\prime}$ and let $\left(x_{1}, y_{1}\right)$ and $\left(x_{2}, y_{2}\right)$ be the coordinates of $v_{1}$ and $v_{2}$ in $p$. coordinates in $q_{\theta}$ are obtained by applying the transformation $R_{\theta}=\left(\begin{array}{cc}\cos \theta & -\sin \theta \\ \sin \theta & \cos \theta\end{array}\right)$, so we have

$$
\begin{gathered}
s_{q_{0}}(e)=s_{p}(e)=\frac{y_{1}-y_{2}}{x_{1}-x_{2}} \text { and } s_{q_{\theta}}(e)=\frac{\left(x_{1}-x_{2}\right) \sin \theta+\left(y_{1}-y_{2}\right) \cos \theta}{\left(x_{1}-x_{2}\right) \cos \theta-\left(y_{1}-y_{2}\right) \sin \theta}, \text { so } \\
s_{q_{\theta}}(e)=r\left(s_{p}(e), \tan \theta\right), \text { where } r(s, t)=\frac{t+s}{1-s t} .
\end{gathered}
$$

Consider any non-zero polynomial $z$ with rational coefficients and $\left|D-e_{0}\right|+$ $\left|D^{\prime}\right|$ variables, labeled as $\mathbf{s}=\left(s_{e}: e \in D-e_{0}\right)$ and $\mathbf{s}^{\prime}=\left(s_{e}^{\prime}: e \in D^{\prime}\right)$. Substituting $\mathbf{s}=\left(s_{p}(e): e \in D-e_{0}\right)$ and $\mathbf{s}^{\prime}=\left(s_{q_{\theta}}(e): e \in D^{\prime}\right)$ into $z$ gives a rational function $z^{*}$ in $\left(s_{p}(e): e \in\left(D-e_{0}\right) \cup D^{\prime}\right)$ and $\tan \theta$. Note that $z^{*}$ is not identically zero, as it is non-zero when $\theta=0$ by the hypothesis that $p$ is quasi-generic. Thus there are only a finite number of values of $\theta \in[0,2 \pi)$ for which $z^{*}$ is zero. Furthermore, the number of such polynomials $z$ is countable, so there are only countably many $\theta$ for which $\left\{s_{p}(e)\right\}_{e \in D-e_{0}} \cup\left\{s_{q_{\theta}}(e)\right\}_{e \in D^{\prime}}$ is algebraically dependent over $\mathbb{Q}$. Thus $B \backslash B^{*}$ is countable, so in particular $B^{*}$ is a dense subset of $B$.

For each $q_{\theta} \in B^{*}$, we can apply Lemma 3.2 to obtain a configuration $p_{\theta}$ : $V \rightarrow \mathbb{R}^{2}$ such that $l_{p_{\theta}}\left(e_{1}\right)=l_{p}\left(e_{1}\right)$, where $e_{1}$ is the unique length edge of $G^{\prime}$, $p_{\theta}(u)=(0,0), s_{p_{\theta}}(e)=s_{p}(e)$ for $e \in D-e_{0}$ and $s_{p_{\theta}}(e)=s_{q_{\theta}}(e)$ for $e \in D^{\prime}$. Since $\left(H^{\prime}, q_{\theta}\right)$ is globally rigid we have $\left.p_{\theta}\right|_{U} \in\left\{q_{\theta},-q_{\theta}\right\}$. Hence $\left(G-e_{0}, p_{\theta}\right)$ is equivalent to $\left(G-e_{0}, p\right)$. Replacing $p_{\theta}$ by $-p_{\theta}$ if necessary, we may suppose that $\left.p_{\theta}\right|_{U}=q_{\theta}$; this determines $p_{\theta}$ uniquely by Lemma 3.2. Now note that the defining conditions of $p_{\theta}$ are polynomial equations with coefficients that are continuous functions of $\theta$, except at a finite set of exceptional values for $\theta$ corresponding to vertical edges in $p_{\theta}$. Since $B^{*}$ is a dense subset of $B$, it follows that $\left\{p_{\theta}: q_{\theta} \in B^{*}\right\}$ all belong to the same component of the framework 
space $S_{G-e_{0}, p, u}$, which is $C$, since $q_{0}=\left.p\right|_{U} \in B^{*}$. Now note that $q_{\pi} \in B^{*}$, as $s_{q_{\pi}}(e)=-s_{p}(e)$ for $e \in D^{\prime}$, so $\left\{s_{p}(e)\right\}_{e \in D-e_{0}} \cup\left\{s_{q_{\pi}}(e)\right\}_{e \in D^{\prime}}$ generates the same extension of $\mathbb{Q}$ as $\left\{s_{p}(e)\right\}_{e \in D-e_{0}} \cup\left\{s_{p}(e)\right\}_{e \in D^{\prime}}$. Therefore $p_{\pi} \in C$. Since $p_{\pi}=-p$ by the uniqueness property noted above, $-p \in C$.

Theorem 9.4. Let $(G, p)$ be a globally rigid generic realisation of a direction irreducible mixed graph $G=(V ; D, L)$ with at least two length edges. Suppose that $L$ induces a length rigid subgraph of $G$. Then $G$ is redundantly rigid.

Proof. We proceed by contradiction. Suppose $G$ is not redundantly rigid. Since $G$ is direction irreducible, Lemma 5.1, implies that $G-e$ is bounded and $r(G-$ $e)=r(G)-1$ for all $e \in D$.

Let $H=(U ; \emptyset, L)$ be the length-rigid subgraph of $G$ induced by $L$. Choose $u \in U$ and $e_{0} \in D$. By translation we can replace the assumption that $(G, p)$ is generic by the assumption that $(G, p)$ is quasi-generic and $p(u)=(0,0)$. Let $H^{\prime}=\left(U ; D^{\prime}, L^{\prime}\right)$ be a minimally rigid graph on the same vertex set as $H$ with exactly one length edge, $f$, and let $G^{\prime}$ be obtained from $G$ by substituting $H$ by $H^{\prime}$. We can show that $G^{\prime}$ is minimally rigid as in the proof of Lemma 9.3.

Let $\left(H^{\prime}, q\right)$ be obtained from $\left(H^{\prime},\left.p\right|_{U}\right)$ by reflection in the $x$-axis. Then $s_{q}(e)=-s_{p}(e)$ for all $e \in D^{\prime}$. Since $\left\{s_{p}(e)\right\}_{e \in D-e_{0}} \cup\left\{s_{p}(e)\right\}_{e \in D^{\prime}}$ is generic, $\left\{s_{p}(e)\right\}_{e \in D-e_{0}} \cup\left\{s_{q}(e)\right\}_{e \in D^{\prime}}$ is generic. Thus we can apply Lemma 3.2 to obtain $p^{\prime}: V \rightarrow \mathbb{R}^{2}$ such that $l_{p^{\prime}}(f)=l_{p}(f), p^{\prime}(v)=(0,0), s_{p^{\prime}}(e)=s_{p}(e)$ for $e \in D-e_{0}$ and $s_{p^{\prime}}(e)=s_{q}(e)$ for $e \in D^{\prime}$. We have $\mathbb{Q}\left(f_{G^{\prime}-e_{0}}\left(p^{\prime}\right)\right)=\mathbb{Q}\left(f_{G^{\prime}-e_{0}}(p)\right)$, so $p^{\prime}$ is quasi-generic by Lemma 2.13. Now consider $\left(G-e_{0}, p^{\prime}\right)$ and let $C$ be the connected component of the framework space $S_{G-e_{0}, p^{\prime}, u}$ which contains $p^{\prime}$. By Lemma 9.3, we have $-p^{\prime} \in C$.

Let $e_{0}=u_{0} v_{0}$. For any $p^{\prime \prime} \in C$ let $F\left(p^{\prime \prime}\right)=\left(p^{\prime \prime}\left(u_{0}\right)-p^{\prime \prime}\left(v_{0}\right)\right) / \| p^{\prime \prime}\left(u_{0}\right)-$ $p^{\prime \prime}\left(v_{0}\right) \|$ be the unit vector in the direction of $p^{\prime \prime}\left(u_{0}\right)-p^{\prime \prime}\left(v_{0}\right)$; this is well-defined since we never have $p^{\prime \prime}\left(u_{0}\right)=p^{\prime \prime}\left(v_{0}\right)$ by [7, Lemma 3.4]. Consider a path $P$ in $C$ from $p^{\prime}$ to $-p^{\prime}$. Then $F\left(p^{\prime \prime}\right)$ changes continuously from $F\left(p^{\prime}\right)$ to $-F\left(p^{\prime}\right)$ along $P$. By the intermediate value theorem there must be some $p^{\prime \prime} \in P$ such that $F\left(p^{\prime \prime}\right)$ is either $F(p)$ or $-F(p)$. Then $\left(G, p^{\prime \prime}\right)$ is equivalent to $(G, p)$. On the other hand $p^{\prime \prime}$ is not congruent to $p$ since $\left.p^{\prime \prime}\right|_{U}$ is obtained from $\left.p\right|_{U}$ by a reflection (as well as a translation and a rotation). It follows that $(G, p)$ is not globally rigid.

Theorem 9.4 shows that if a graph satisfies the hypotheses of Theorem 6.1, and satisfies the additional hypothesis that its length edges induce a lengthrigid subgraph, then generic realisations of this graph are never globally rigid. This supports Conjectures 9.1 and 9.2. We close by noting that Lemma 5.1 and Theorem 9.4 imply that the mixed graph shown in Figure 3 is the smallest graph for which these conjectures are not known to be true. 


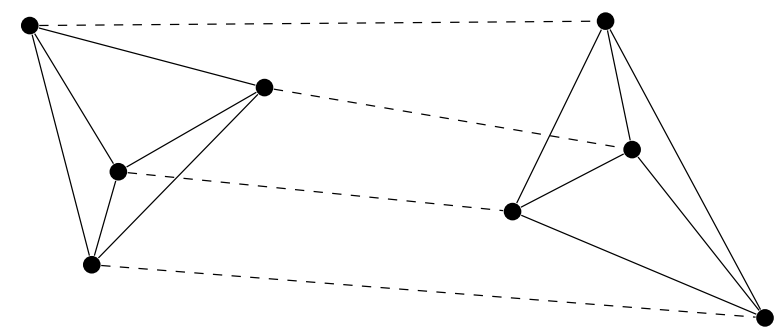

Figure 3: A direction irreducible mixed graph which is not redundantly rigid. We know it has a generic realisation which is not globally rigid by Theorem 1.5, but do not know whether all generic realisation have this property.

\section{References}

[1] A. Berg And T. Jordán, Algorithms for graph rigidity and scene analysis, in: Proceedings of the 11th Annual European Symposium on Algorithms 2003, Springer Lecture Notes in Computer Science, vol. 2832, 2003, 78-89.

[2] K. Clinch, Global rigidity of 2-dimensional direction-length frameworks with connected rigidity matroids, arXiv:1608.08559v1 [math.CO]

[3] H.N. Gabow, Algorithms for graphic polymatroids and parametric $\bar{s}$-sets, J. Algorithms 26 (1998), no. 1, 48-86.

[4] J.E. Hopcroft, R.E. TARJan, Dividing a graph into triconnected components, SIAM J. Comput. 2 (1973), 135-158.

[5] B. JaCkson And T. Jordán, Connected rigidity matroids and unique realisations of graphs, J. Combin. Theory Ser. B 94 (2005) 1-29.

[6] B. JACKSON AND T. Jordán, Globally rigid circuits of the directionlength rigidity matroid, J. Combin. Theory Ser. B 100 (2010) 1-22.

[7] B. JACKSON AND T. JORDÁN, Operations preserving global rigidity of generic direction-length frameworks, International Journal of Computational Geometry and Applications 20 (2010) 685-708.

[8] B. Jackson and P. Keevash, Bounded direction-length frameworks, Discrete and Computational Geometry 46 (2011) 46-71.

[9] B. JaCkson and P. KeEvash, Necessary conditions for global rigidity of direction-length frameworks, Discrete and Computational Geometry 46 (2011), 72-85.

[10] G. Laman, On graphs and rigidity of plane skeletal structures, J. Engineering Math. 4 (1970), 331-340.

[11] A. Lee and I. Streinu, Pebble game algorithms and sparse graphs, Discrete Math. 308 (2008) 1425-1437. 
[12] C.St.J.A. Nash-Williams, Decomposition of finite graphs into forests, J. London Math. Soc. 39 (1964), 12.

[13] J. OxLEY, Matroid Theory, Oxford University Press, 1992.

[14] B. Servatius and W. Whiteley, Constraining plane configurations in CAD: Combinatorics of directions and lengths, SIAM J. Disc. Math. 12 (1999), 136-153.

[15] W. Whiteley, The union of matroids and the rigidity of frameworks, SIAM J. Disc. Math. 1 (1988), 237-255.

[16] W. Whiteley Some matroids from discrete applied geometry, in: Matroid Theory, AMS Contemporary Mathematics, vol. 197, (1996), 171-313. 